\section{MIRD Monograph: Radiobiology and Dosimetry for Radiopharmaceutical Therapy with Alpha- Particle Emitters}

\author{
G. Sgouros, Ed.
}

Reston, VA: Society of Nuclear Medicine and Molecular Imaging, 2015, 67 pages, $\$ 49$

Publication of MIRD Monograph: Radiobiology and Dosimetry for Radiopharmaceutical Therapy with Alpha-Particle Emitters was supported by the SNMMI MIRD Committee to provide clinicians with information on radiopharmaceutical therapies that use $\alpha$-particle emitters. George Sgouros, the MIRD committee chair, stated that the objective of this book is to "provide guidance and recommendations for human dosimetry" in these therapies.

The book begins with an introduction to $\alpha$-particles and an excellent historical overview of the use of radioactivity and $\alpha$-emitters since 1896 , followed by an explanation of the radiobiology of $\alpha$-particles (how they work) and relative biological effectiveness (what the radiation dose to patients might be).

In a section covering radiation dosimetry, readers are told how $\alpha$-particles affect each part of the body, including the bone marrow and normal organs, as well as the effects at the cellular level. This part of the book can help guide clinicians in tailoring radiopharmaceutical therapy to a specific patient. Challenges concerning detection of $\alpha$-particles are then discussed, with an important piece of the puzzle being an understanding of the instruments capable of detecting $\alpha$-particles.

It is also important to understand how the different $\alpha$-particleemitting tracers are delivered, and the book includes a valuable section detailing the different methods of localization.
The next section comprehensively details $\alpha$-particle emitters that either are already in human use or have a possibility of entering human use. An overview is provided of each of $6 \alpha$-particle-emitting radionuclides, their properties, and the preclinical and clinical studies in which they are being used.

The section on dosimetry recommendations thoroughly explains the importance of using dosimetric measurements. $\alpha$-therapies can be highly effective but also extremely toxic. Understanding how dosimetry can be used to accurately determine dose is essential.

The book ends with radiation safety and regulatory considerations. Radiation safety is not as great a concern as potential contamination. Regulatory requirements and licensure must be met, and this section briefly touches on both topics.

Although comprising only about 60 pages, the book contains all the information a clinician needs to better understand radiopharmaceutical therapies with $\alpha$-particle emitters. Technologists can also gain an understanding of $\alpha$-particle emitters, but the book is geared more toward clinicians.

The use of charts, graphs, and pictures helps the reader understand the material, and more than 500 publications are referenced. I believe that the book has met its stated objective and should be considered by anyone wanting to learn more about $\alpha$-particle emitters.

Ellie Mantel, CNMT, RT(N), FSNMMI-TS

Hospital of the University of Pennsylvania 3400 Spruce St., 3 Donner Philadelphia, PA 19104 eleanor.mantel@uphs.upenn.edu

Published online Mar. 10, 2016. DOI: 10.2967/jnmt.116.175034 